\title{
Modeling of Biological Intelligence for SCM System Optimization
}

\author{
Shengyong Chen, ${ }^{1}$ Yujun Zheng, ${ }^{1}$ Carlo Cattani, ${ }^{2}$ and Wanliang Wang ${ }^{1}$ \\ ${ }^{1}$ College of Computer Science \& Technology, Zhejiang University of Technology, Hangzhou 310023, China \\ ${ }^{2}$ Department of Mathematics, University of Salerno, Via Ponte Don Melillo, 84084 Fisciano, Italy
}

Correspondence should be addressed to Shengyong Chen, sy@ieee.org

Received 15 September 2011; Accepted 15 October 2011

Academic Editor: Maria Crisan

Copyright (C) 2012 Shengyong Chen et al. This is an open access article distributed under the Creative Commons Attribution License, which permits unrestricted use, distribution, and reproduction in any medium, provided the original work is properly cited.

This article summarizes some methods from biological intelligence for modeling and optimization of supply chain management (SCM) systems, including genetic algorithms, evolutionary programming, differential evolution, swarm intelligence, artificial immune, and other biological intelligence related methods. An SCM system is adaptive, dynamic, open self-organizing, which is maintained by flows of information, materials, goods, funds, and energy. Traditional methods for modeling and optimizing complex SCM systems require huge amounts of computing resources, and biological intelligence-based solutions can often provide valuable alternatives for efficiently solving problems. The paper summarizes the recent related methods for the design and optimization of SCM systems, which covers the most widely used genetic algorithms and other evolutionary algorithms.

\section{Introduction}

Supply chains are a kind of network with facilities and distribution entities (suppliers, manufacturers, distributors, retailers). The supply chain performs the functions of procurement of raw materials, transformation of raw materials into intermediate and finished products, and distribution of finished products to customers [1]. Due to rising product and market complexity, expanding competition, shorter product lifecycles, and changing customer demands, today's supply chain management (SCM) systems increasingly involve complex sets of objectives and constraints, and variations of uncertainty and randomization [2], and thus static and/or centralized models are insufficient to effectively plan, coordinate, and optimize activities in a supply chain.

Modeling and optimization of an SCM system provide a critical support for decision making in a competitive market. According to [3], the basic approaches to supply chain modeling and optimization can be divided into five classes:

(1) fundamental formulation of supply chains,

(2) integer-mixed programming optimization,

(3) stochastic programming,
(4) heuristic methods,

(5) simulation-based methods.

Traditional simulation methods for large-scale complex systems require huge amounts of computing resources [4]. In recent years, bioinspired methods have gained increasing interest in the research of modeling and optimization for SCM systems which are typically dynamic, open selforganizing systems maintained by flows of information, materials, goods, funds, and energy. Bioinspired and living system mechanisms, such as learning, growth, evolution, collaboration, and competition, bring an innovative solution for the analysis and improvement of emergent complex behaviors in virtually computing modules. In this paper, we review the recent major accomplishments in bioinspired solution methods and tools for SCM systems. In particular, we concentrate on the modeling and optimization methods based on a class of metaheuristics inspired by biologically living beings, including genetic algorithms (GAs) [5], evolutionary programming (EP) [6], evolution strategies (ESs) $[7,8]$ and differential evolution (DE) [9], swarm intelligence $[10,11]$, and artificial immune [12]. These heuristic methods usually do not require deep mathematical knowledge, and have been demonstrated to be quite useful and efficient 
in optimization search for large-scale problems. We believe that this work will help researchers and practitioners to gain knowledge about the major developments emerged throughout the years and find valuable approaches that can be referred in the research or applied in the practice of SCM modeling and optimization.

The rest of the paper is synthesized as follows: Section 2 describes modeling and optimization methods based on GA, Section 3 depicts three other evolution-related methods including EP, ES, and DE, Section 4 describes methods based on swarm intelligence, and Section 5 introduces some other biological methods. Finally we discuss some future trends in Section 6 and conclude in Section 7.

\section{Genetic Algorithms}

2.1. Design of Supply Chain Network. Network design plays a key role in achieving efficient and effective management of SCM systems. Typically, a supply chain can be represented as a form of multistage-based structure, the optimal design of which has been recognized as NP-hard problems that combine the multiple choice knapsack problem with the capacitated location-allocation problem [13]. The first attempt to use the GA approach to solve the SCM network design problems has been proposed by Zhou et al. [14]. They developed a balanced star-spanning forest formulation for encoding the solutions and then used uniform crossover and exchange mutation operators in the algorithm. The experiment showed that for a maximum of 10 distributors and 100 customers, the algorithm can balanced all the distributors. Gen et al. [15] proposed a set of spanning tree-based for a class of network design problems such as degree-constrained minimum spanning tree problems, capacitated minimum spanning tree problems, fixed charge transportation problems, and network topological design problems, which were applied to some real-world SCM systems.

A multistage distribution problem is a standard one with supply chain network design. Many works focus on the twostage supply chain distribution problem [16-20], which can be represented in Figure 1. That is, each of the $m$ plants can ship to any of the $n$ distribution centers, and each of the $n$ distribution centers can ship to any of the $p$ customers. Typically, each plant $i$ has $s_{i}$ units of supply, each customer $k$ has $d_{k}$ units of demand, and each distribution centre $j$ has $t_{j}$ units of stocking capacity, and the main purpose is to minimize the total cost which may include transportation cost, fixed cost, and transportation time.

Aiming at the demand allocation optimization of a two-stage and single product supply chain design problem, Chan et al. [18] implemented a multicriteria optimization algorithm which combines GA with the decision-making technique of the Analytic Hierarchy Process (AHP). Jawahar and Balaji [19] considered a two-stage distribution problem of a supply chain that is associated with a fixed charge and presented a GA that evolves the solution for best fitness of total cost of distribution. By fine-tuning its parameters, the algorithm can work out optimal solutions for smallsize problem instances, but its performance on large-size

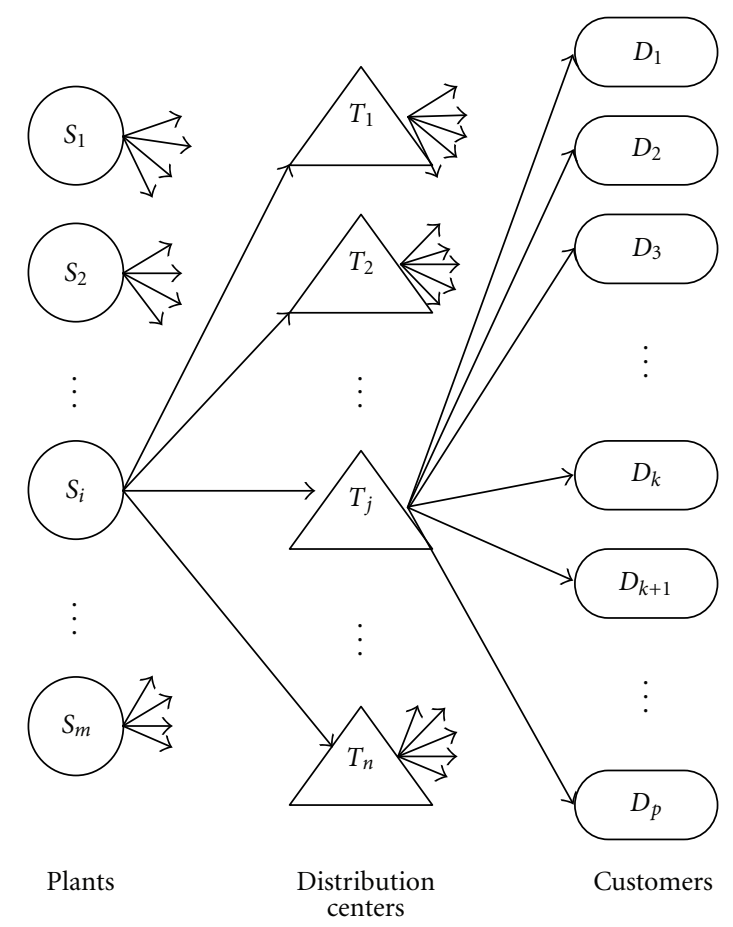

FIGURE 1: Illustration of the two-stage supply chain network design problems.

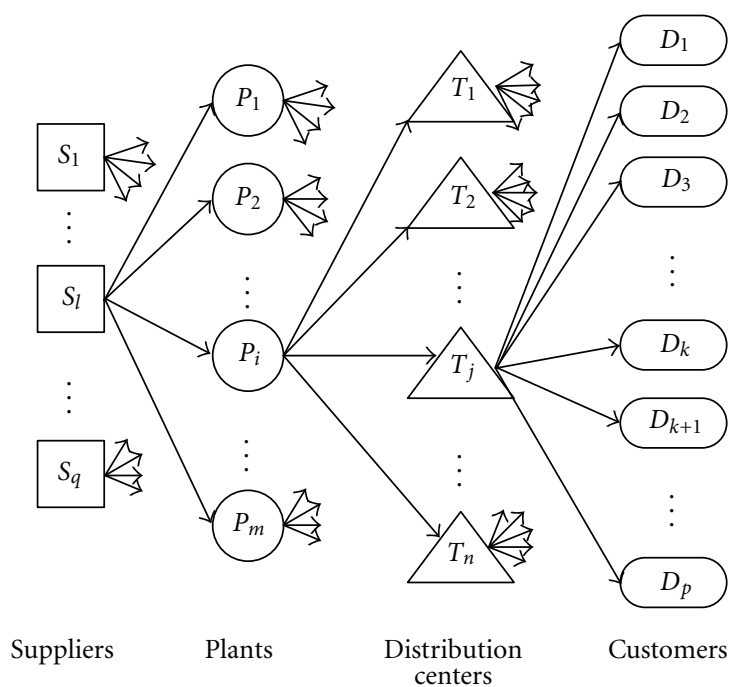

FIGURE 2: Design problem in three-stage supply chain networks.

instances has not been demonstrated. Feng and Zhang [20] extended the problem by involving multiple transportation modes and proposed a GA that can deal with middle-size problem instances.

Since that today's market environment becomes more and more complex, supply chains with multiple (three or more) stages are common. To model a single product three-stage supply chain network (as illustrated in Figure 2), Altiparmak et al. [21] proposed a GA that uses a threesegment chromosome string which is decoded through 
a backward procedure from the third stage up to the first stage connecting suppliers and plants. They also extended their priority-based encoding in a new GA for the multiproduct case in [22]. However, their decoding structure can require some repair procedures when the upper limits are exceeded; otherwise the capacities will be not enough to meet the customers' demand. Costa et al. [23] presented a new chromosome encoding and a complementary decoding procedure able to overcome the drawbacks and thus improve the efficiency and effectiveness of three-stage supply chains.

Considering that in a multistage supply chain network the flow can be only transferred between two consecutive stages, Yeh [24] presented a memetic algorithm for the design optimization of distribution network, which combined GA, linear programming, a greedy-heuristic based method, and three local search methods. Yun et al. [25] indicated that, when applying GA to multistage-based supply chains, conventional GA can do global search but there is no way for local search around the convergence area generate, and they proposed a new hybrid GA with an adaptive local search scheme which can automatically control whether local search technique is used in GA loop or not, and thus can reliably solve various optimization problems without trial-and-error experimentation.

2.2. Supply Chain Planning and Scheduling. The success of SCM highly depends on the timely and efficient production/distribution in the supply chain network, which can be typically regarded as a combination of planning and scheduling problems, each seriously affected by nearly prohibitive combinatorial complexity. In general, the development of an effective approach for SCM planning and scheduling can be divided into two steps:

(1) developing a precise mathematical model of the problem considered, explaining the objective function(s), decision variables, and problem constraints;

(2) selecting one or more effective metaheuristics suited to the problem, and developing a problem-solving algorithm-based on the heuristics.

Şerifoğlu and Ulusoy [26] considered the problem of scheduling independent jobs on identical parallel machines to minimize earliness/tardiness, which incorporates distinct due dates and arrival times for jobs, different processing rates for machines, and sequence-dependent set-up times into the problem formulation. They developed a GA with a new multicomponent uniform order-based crossover (MCUOX) operator and demonstrated that the GA outperforms other simple heuristic methods especially in larger-sized problems. Min and Cheng [27] also proposed a GA for solving the problem of scheduling identical parallel machines, where the objective is formulated as the minimization of the makespan.

Garcia et al. [28] considered the problem of scheduling a single production plant in order to satisfy delivery time constraints. They proposed two approaches, an exact method for simple cases and a GA for instances of more realistic size. Feng et al. [29] considered the problem of scheduling a single depot equipped with a fleet of vehicles with identical capacity and fixed loading/unloading times. They proposed a GA for searching a production sequence maximizing a predefined performance index, taking truck waiting times and a penalty for violating the unloading continuity of multitruck orders into consideration.

In [30], Lee et al. studied an operation-level advance planning and scheduling problem in supply chain, the model of which is to determine the best schedule using alternative operation sequences and machines, considering scheduling with outsourcing and strong constraints of the due dates of customer orders. They developed a genetic algorithm-based heuristic to solve it. Karabuk [31] studied the assignment of jobs to suppliers and aims to determine optimal production scheduling that minimizes the makespan, where each supplier requires a different length of time to process each job. The research proposed an adaptive GA with a new dominated gene crossover operator to solve this problem. Zegordi et al. [32] considered the scheduling of products and vehicles in a two-stage supply chain environment, assuming that the various output products occupy different percentages of each vehicle's capacity and proposed a gendered GA with two different chromosomes with nonequivalent structures that performs better than standard GA with a unique chromosomal structure.

There are also a number of reports on the applications of genetic algorithm in different trades, for example, [33-40]. Considering a semiconductor supply chain for reflecting the nonlinear throughput time of manufacturing, Chidambaram and Armbruster [33] proposed a hybrid LP and GA framework to solve the non-linear programming problem in order to avoid significant differences between the planned and realized output. Naso et al. [34] considered the problem of finding an optimized schedule for the justin-time production and delivery of ready-mixed concrete on a set of distributed and coordinated production centers. They proposed a hybrid evolutionary algorithm in which the GA constitutes the core of the search strategy, while multiple heuristic rules called in specific circumstances contribute to reconstruct a feasible solution that satisfies all the constraints and objectives. The algorithm can guarantee the determination of a feasible schedule for any given set of requests and can address the highly complex scheduling problem of an entire supply-chain for just-in-time production. Dong and Ding [35] introduced a dynamic berth allocation model for container terminal and proposed a GAbased heuristic method that improves the existing research on static berth allocation models. The GA-based approach explores detailed capabilities of a complex problem solution using these two encoding methods. Recently, Delavar et al. [36] considered coordinated scheduling of production and air transportation and proposed two GA approaches to optimize customer service at minimum total cost: the first uses a portion of the search time to seek for the best transportation allocation and dedicates the remaining time to search for the best production-transportation solution; the seconds allocates some definite number of generations to search for the best production-transportation solution only after finding a better solution in each generation of transportation. In both algorithms, the Taguchi parameter design method was employed to adjust the parameters. 
2.3. Multiobjective Optimization. In most cases, the design, planning, and scheduling of complex supply chains will involve trade-offs among different goals. Since 2000, multiobjective optimization of SCM systems has gained a lot of interests of the researchers. Generally, for a multiobjective optimization problem we need to search for a set of Pareto optimal solutions rather than a single optimal one. Using a minimization problem for illustration, let $f_{1}, f_{2}, \ldots, f_{m}$ be objective functions; a solution $\mathbf{x}$ is said to dominate $\mathbf{y}$ if and only if

(i) $f_{i}(\mathbf{x}) \leq f_{i}(\mathbf{y})$ for all $i \in\{1,2, \ldots, m\}$,

(ii) $f_{i}(\mathbf{x})<f_{i}(\mathbf{y})$ for existing $i \in\{1,2, \ldots, m\}$.

Regarding this, a Pareto-optimal front of the problem consists of all solutions for which the corresponding objective vectors cannot be improved in a given dimension without worsening another [41].

A typical multiobjective genetic optimization algorithm was proposed by Chan and Chung [42] for simultaneously minimizing the total cost of the system, total delivery days, and the equity of the capacity utilization ratio for manufacturers. In [43] Chan et al. proposed a hybrid GA for production and distribution problem, which utilized AHP to construct these criteria and calculate the fitness value of chromosome, and considered operating cost, service level, and resources utilization as objectives. Considering that all organizational units that participate on a single SC network are distributed by nature, constrained, and self-interested, Al-Mutawah et al. [44] used a distributed multiobjective GA to solve a three-subchain optimization problem, and their test results showed that the distributed GA provided an improved computational performance, because realworld supply chain applications are distributed in naturedistributed approach.

Altiparmak et al. [45] considered SCM optimization problem with three objectives: minimizing total cost, maximizing of customer services, and maximizing capacity utilization balance for distribution centers. To deal with the objectives and enable the decision maker for evaluating a number of alternative solutions, they proposed a GA which was designed to generate Pareto-optimal solutions considering two different weight approaches. Farahani and Elahipanah [46] adopted a hybrid non-dominated sorting GA to optimize total cost and service level for JIT distribution in a supply chain, whose results were compared with Lingo software to evaluate the performance of proposed algorithm. Che and Chiang [47] established a multiobjective optimization mathematical model for the build-to-order supply chain model which are defined as "the system that produces goods and services based on individual customer requirements in a timely and cost competitive manner by leveraging global outsourcing, the application of information technology and through the standardization of components and delayed product differentiation strategies" [48]. Considering three evaluation criteria including costs, delivery time, and quality, they proposed a modified Pareto GA to improve efficiency of the crossover and mutation operators of basic Pareto GA.

\section{EP, ES, and DE}

Besides GA, other evolutionary algorithmic methods have also been applied to many SCM modeling and optimization problems. EP was devised in order to evolve finite state machines for the prediction of events on the basis of former observations and has been demonstrated useful for searching the optimum of nonlinear functions [49]. Huang and $\mathrm{Lu}$ [50] proposed an interactive EP approach based on the relativistic error and selection of some other parameters to improve initial value determination, mutation, and variance parameter selective operation. The simulation results of supply chains showed that the improved EP is much more appropriate of nonline model with a great volume of data. Based on EP approach, Li et al. [51] proposed a heuristic strategic safety stock optimization algorithm for reverse logistics SCM considering the modeling complexity of external as well as internal product returns and reuses of supply chains.

Original ES uses a mutation operator that produces a single descendent from a given ancestor $[7,8]$, denominated ES- $(1+1)$, and was progressively generalized to ES- $(\mu+\lambda)$, that is, several ancestors $(\mu>1)$ and descendents $(\lambda>1)$ in each generation. Homberger [52] proposed an approach that combines the $(1+\lambda)$-selection procedure with the Borda maximin voting rule, to coordinate decentral planning of a group of independent and self-interested decision makers, who are searching for an agreeable contract regarding multiple interdependent issues, in the case of asymmetric information presented. In [53] Dalkilic et al. developed an ES algorithm to solve a multiple-supplier multiple-item problem with stochastic lead times, which was successfully applied to some real-world healthcare SCM cases.

DE approach combines simple arithmetic operators with the classical operators of crossover, mutation, and selection to evolve a randomly generated starting population to a final solution. It is similar to a $(\mu+\lambda) \mathrm{ES}$, but in DE the mutation is not done via some separately defined probability density function [54]. Routroy and Kodali [55] developed a DE algorithm for minimizing the total systemwide cost, which consists of supply chain inventory capital, supply chain ordering/set-up cost, and supply chain stock-out cost. The result showed that the algorithm helps in determining ordering/production quantity and inventory/service level that should be maintained by each member of the supply chain. The algorithm was further extended for multiechelon supply chain inventory problems [56] and the problems with demand and leadtime uncertainty [57]. Prasertwattana and Shimizu also [58] applied a similar DE algorithm to optimize material ordering and inventory control of SCM systems.

DE was used for multiobjective optimization of SCM systems first by Babu and Gujarathi [59]. In their algorithm, crossover is carried out between the target vector and the noisy random vector to generate a trial vector, the cost of trial and target vectors is compared, and the variables corresponding to best cost are passed into next generation. The algorithm was successfully applied to a three-stage SCM problem. Dos Santos Coelho and Lopes [60] firstly developed a chaotic DE algorithm for the optimization of 
a supply chain, which was based on different DE approaches combined with chaotic sequences and led to better results than basic DE approaches. In [61] Xu et al. proposed a migration $\mathrm{DE}$ algorithm by imitating nomadic migration for this supply chain problem and presented an ensemble method based on different DE methods for not only avoiding the premature convergence but also improving the global search capability.

Falcone et al. [54] compared the performance of GA, EP, ES, and DE based on a case of integrated productioninventory-distribution SCM system. Their results showed that the robustness of the evolutionary methods is in general, and the efficiency of DE, in particular, suggests their great utility for the supply chain optimization problem.

\section{Swarm Intelligence}

4.1. Ant Colony Optimization. Ant colony optimization (ACO) algorithm mimics the behavior of real ants living in colonies that communicate with each other using pheromones in order to accomplish complex tasks such as establishing a shortest path from the nest to food sources [62]. Silva et al. [63] proposed an ACO algorithm for distributed optimization of a logistic system, but the work only considered only the allocation of suppliers in the system. In a successive work [64], the authors modeled a distributed optimization problem for a generic supply chain with suppliers, logistics, and distributers and developed an ACO algorithm that allows the exchange of information between different optimization problems by means of a pheromone matrix. The experimental results showed that the approach can significantly improve global supply chain performance with respect to other simple decentralized approaches.

Wang [65] studied the partner selection and productiondistribution planning problem in a supply chain with the losses of production, which is called the defective supply chain. He developed for this problem a two-phase ACO algorithm, which finds out the combination of the maximum yield rate and the minimum number of partners in the first phase and implements the distribution with the partners and seeks out the minimum value of T-score in the second phase. Comparative numerical experiment showed that his algorithm achieves better performance than the common single-phase ACO algorithms.

In a very recent work, Moncayo-Martínez and Zhang [66] studied the multiobjective ACO for supply chain optimization. They considered the problem for minimizing the total cost while keeping the total lead-times within required delivery due dates. They formulated the design problem into an ACO optimization form and implemented a number of ant colonies in a sequence to explore the solution space and search for successively better nondominated set of supply chain designs.

4.2. Particle Swarm Optimization. Particle swarm optimization (PSO) [10] is another population-based global optimization technique that enables a number of individual solutions, called particles, to move through a hyperdimensional search space to search for the optimum. Each par- ticle has a position vector and a velocity vector, which are adjusted at iterations by learning from a local best found by the particle itself and a current global best found by the whole swarm. Modeling a system where multiplecandidate solution coexists and collaborates simultaneously, PSO approaches embed problem-solving attempts in a social network and are suitable in nature for the optimization of very complex systems [67] and thus have been successfully applied in the research of SCM, for example, [68-74].

Izquierdo et al. [68] applied a PSO algorithm to a supply chain for searching optimal biomass flows from sources to energy production plants. Kadadevaramath et al. [70] proposed a PSO algorithm for the modeling and optimization of a four-stage supply chain and gained satisfying results. Bachlaus et al. [71] considered the design of a multiechelon supply chain network that integrates production, distribution and logistics activities and developed a hybrid PSO algorithm based on Taguchi robust design optimization tool [75]. The algorithm incorporates the characteristics of statistical design of experiments and random search techniques, which is an attractive way for determining flexible location and distribution strategies.

In [73] Sinha et al. considered the optimization of resource allocation for agents in a petroleum supply chain. They developed a coevolutionary PSO algorithm with two populations, and the decision vectors and the Lagrangian multipliers are taken to be constant in the first population and to be variables in the second population. The algorithm also uses a Cauchy random number distribution which is proved to be much better than a Gaussian distribution.

Soares [76] et al. utilized four kinds algorithms (including EP, GA, PSO, and EDA-estimation of distribution algorithm) to solve a multiple-retailer SCM problem, which is for finding an optimal balance of quantities ordered from suppliers and acceptable lead time costs while taking into account limiting factors such as the time each retailer will wait for a backorder. According to the results on the testsuite, three PSO algorithms of the 32 attempted algorithms demonstrate great flexibility and high performance.

4.3. Artificial Bee Colony Optimization. There are several optimization algorithms [77-80] that simulate the intelligent foraging behavior of a honeybee swarm. A more recent and popular approach is the artificial bee colony $(\mathrm{ABC})$ algorithm that divides a bee colony into three groups, namely, employed bees exploiting on current food sources, onlookers waiting in the hive for choosing existing food sources, and scouts bees exploring new food sources. In [81] Kumar et al. analyzed the complexities of a remanufacturing problem in which the return rate is a function of environmental factor and proposed an $\mathrm{ABC}$ algorithm for the problem model. In their test results, the $A B C$ algorithm significantly outperformed a PSO algorithm for comparison. In [82], Pal et al. considered a problem of integrated procurement, production, and shipment planning for a three-echelon supply chain. They developed two ABC-based algorithms for optimizing the order scheduling and production-shipment planning to achieve a minimum cost. 
Considering the problem of a milk production, scheduling, and supply network design with extensively multiple conflicting objectives, Banerjee et al. [83] proposed a Pareto BCO approach, which was demonstrated to be better than some other bioinspired algorithms by simulation and comparison. For improving machine utilization and reducing cycle-time in manufacturing industry, Li et al. [84] applied a Pareto bee colony optimization (BCO) algorithm for a multiobjective flexible job shop scheduling problem and gained good computational result.

\section{Other Methods from Biological Intelligence}

Provoked by the theoretical immunology, observed immune functions, principles and models, artificial immune system (AIS) stimulates the adaptive immune system of a living creature to unravel the various complexities in real-world engineering optimization problems [85]. Shukla et al. [86] employed an AIS approach to a batch sequencing problem in a multistage supply chain, which considers three objectives including minimization of lead time, blocking time, and due date violation, and the experimental results showed that the AIS outperforms GA and simulated annealing (SA).

Prakash and Deshmukh [85] considered a multiple vendor transportation problem with time and cost criteria and proposed an AIS algorithm strengthened by a fuzzy logic controller (FLC) to solve the multicriteria problem. AIS works as an evolutionary search algorithm to find out the Pareto optimal front, whereas FLC is implemented to change the hypermutation rate adaptively on the basis of the fitness values at each iteration. They also employed a web-based supply chain to facilitate the SCM enterprise by e-learning.

In [87] Hajiaghaei-Keshteli considered a two-stage supply chain network of distribution centers and customers. To solve the problem for selecting some potential places as distribution centers in order to supply demands of all customers with minimum opening cost plus shipping cost, he, respectively, developed a GA and an AIS algorithm, and the results showed that the AIS algorithm exhibits robust performance improvements in large size problems versus GA.

\section{Discussion}

We have summarized the main bioinspired methods for SCM system design and optimization. It is deserved to note that swarm-based methods and artificial immune systems are not yet mature and thus are expected to gain more research interests. With the increasing importance and complexity of SCM systems, researchers are facing the challenges to promote the performance, reliability, and scalability of SCM problem-solving methods, and here we highlight the following future trends in bioinspired computation in SCM systems.

6.1. Hybrid. Different bioinspired methods have different design principles and application areas. As we mentioned in previous sections, there are a number of studies that exploit the strengths of several individual methods to obtain a more powerful approach to dealing with complex SCM problems, and to a great extent, these hybrids methods are shown to be more competitive than individual methods. It can be anticipated that future research will continuously put great emphasis on the hybridization of bioinspired methods, for example, swarm-based evolutionary algorithms [88-90], and the hybridization of bioinspired methods with other approaches such as local search [91], tabu search [92], and simulated annealing [93].

6.2. Extension with New Computing Paradigms. We are seeing that innovative informational/computational paradigms, such as chaotic systems, quantum informatics, and DNA computing, provide valuable inspiration to create new heuristics for complex optimization problems including a host of NP-hard problems. Thus, the extensions of current bioinspired methods based on these new paradigms are expected to achieve dramatic improvement on computational performance. For example, chaotic sequencing and local search operations have been successfully applied for helping evolutionary algorithms avoiding premature convergence effectively $[94,95]$. Also, quantum-inspired evolutionary algorithms are regarded as one of the three main research areas related to the complex interaction between quantum computing and evolutionary algorithms [96-98] and have been applied in some SCM optimization problems in very recent research [99]. These approaches are expected to show great promises for the future.

\section{Conclusion}

Today's SCM systems have to deal with ever-changing markets and intrinsic structural complexity emerging from virtually infinite number of interacting entities. Therefore, the community requires effective artificial intelligence methods and tools for modeling and optimizing largescale complex supply chains. The paper has reviewed the recent development of bioinspired methods in SCM applications. Typical illustrations are addressed for evolutionary algorithms including GA, EP, ES, and DE, swarm-based intelligent algorithms including ant colony, particle swarm and artificial bee colony, and other bioinspired methods like AIS. Representative works are summarized for helping readers to have a general overview of the state of the art and to easily refer to suitable methods in practical solutions. Over the last decade, bioinspired methods have experienced a rapid growth and have successfully applied to the design and optimization of highly complex systems such as SCM systems. The fruits of these researches are continuously becoming new technological solutions to new open problems, and the full potential is far from being reached.

\section{Acknowledgments}

This work was supported by the National Natural Science Foundation of China (61105073, 61173096, 60874074, 
60870002), Zhejiang Provincial S\&T Department (2010R10006, 2010C33095), and Zhejiang Provincial Natural Science Foundation (R1110679).

\section{References}

[1] H. Sarimveis, P. Patrinos, C. D. Tarantilis, and C. T. Kiranoudis, "Dynamic modeling and control of supply chain systems: a review," Computers and Operations Research, vol. 35, no. 11, pp. 3530-3561, 2008.

[2] Y. Zheng, J. Wang, and J. Xue, "A-team based supply chain management agent architecture," International Journal on Artificial Intelligence Tools, vol. 18, no. 6, pp. 801-823, 2009.

[3] M. Dong, Process modeling, performance analysis and configuration simulation in integrated supply chain network design, Ph.D. thesis, Virginia Polytechnic Institute and State University, Blacksburg, Va, USA, 2001.

[4] E. Sanchez, A. Perez-Uribe, A. Upegui et al., "PERPLEXUS: pervasive computing framework for modeling complex virtually-unbounded systems," in Proceedings of the 2nd NASA/ESA Conference on Adaptive Hardware and Systems (AHS '07), pp. 587-591, August 2007.

[5] J. H. Holland, Adaptation in Natural and Artificial Systems, The University of Michigan Press, Ann Arbor, Mich, USA, 1975.

[6] D. B. Fogel, "Introduction to simulated evolutionary optimization," IEEE Transactions on Neural Networks, vol. 5, no. 1, pp. 3-14, 1994.

[7] I. Rechenberg, Evolutionsstrategie: Optimierung Technischer Systeme Nach Prinzipien der Biologischen Evolution, Frommberg-Holzboog, Stuttgart, Germany, 1973.

[8] H. P. Schwefel, Kybernetischer Evolution als Strategie der experimentellen Forschung in der Strömungstechnik, Diploma thesis, Technical University of Berlin, Berlin, Germany, 1975.

[9] R. Storn and K. Price, "Differential evolution: a simple and efficient adaptive scheme for global optimization over continuous spaces," Tech. Rep. TR-95-012, International Computer Science Institute, Berkeley, Calif, USA, 1995.

[10] J. Kennedy and R. Eberhart, "Particle swarm optimization," in Proceedings of the IEEE International Conference on Neural Networks, pp. 1942-1948, Perth WA, Australia, December 1995.

[11] M. Clerc, Particle Swarm Optimization, ISTE, London, UK, 2006.

[12] J. D. Farmer, N. H. Packard, and A. S. Perelson, "The immune system, adaptation, and machine learning," Physica D: Nonlinear Phenomena, vol. 22, no. 1-3, pp. 187-204, 1986.

[13] M. Gen and R. Cheng, Genetic algorithms and engineering design, Wiley-Interscience, New York, NY, USA, 1997.

[14] G. Zhou, H. Min, and M. Gen, "The balanced allocation of customers to multiple distribution centers in the supply chain network: a genetic algorithm approach," Computers and Industrial Engineering, vol. 43, no. 1-2, pp. 251-261, 2002.

[15] M. Gen, A. Kumar, and J. R. Kim, "Recent network design techniques using evolutionary algorithms," International Journal of Production Economics, vol. 98, no. 2, pp. 251-261, 2005.

[16] A. Cakravastia, I. S. Toha, and N. Nakamura, "A two-stage model for the design of supply chain networks," International Journal of Production Economics, vol. 80, no. 3, pp. 231-248, 2002.
[17] G. Wang, Y. Liu, and M. Zheng, "Fuzzy two-stage supply chain problem and its intelligent algorithm," in Proceedings of the 6th International Symposium on Neural Networks, vol. 5552, part 2 of Lecture Notes in Computer Science, pp. 15-24, Wuhan, China, 2009.

[18] F. T. S. Chan, S. H. Chung, and S. Wadhwa, "A heuristic methodology for order distribution in a demand driven collaborative supply chain," International Journal of Production Research, vol. 42, no. 1, pp. 1-19, 2004.

[19] N. Jawahar and A. N. Balaji, "A genetic algorithm for the twostage supply chain distribution problem associated with a fixed charge," European Journal of Operational Research, vol. 194, no. 2, pp. 496-537, 2009.

[20] C. Feng and Y. Zhang, "A genetic algorithm of two-stage supply chain distribution problem associated with fixed charge and multiple transportation modes," in Proceedings of the 5th International Conference on Natural Computation (ICNC '09), pp. 76-80, Tianjin, China, August 2009.

[21] F. Altiparmak, M. Gen, L. Lin, and T. Paksoy, "A genetic algorithm approach for multi-objective optimization of supply chain networks," Computers and Industrial Engineering, vol. 51, no. 1, pp. 196-215, 2006.

[22] F. Altiparmak, M. Gen, L. Lin, and I. Karaoglan, "A steadystate genetic algorithm for multi-product supply chain network design," Computers and Industrial Engineering, vol. 56, no. 2, pp. 521-537, 2009.

[23] A. Costa, G. Celano, S. Fichera, and E. Trovato, "A new efficient encoding/decoding procedure for the design of a supply chain network with genetic algorithms," Computers and Industrial Engineering, vol. 59, no. 4, pp. 986-999, 2010.

[24] W. C. Yeh, "An efficient memetic algorithm for the multistage supply chain network problem," International Journal of Advanced Manufacturing Technology, vol. 29, no. 7-8, pp. 803813, 2006.

[25] Y. Yun, C. Moon, and D. Kim, "Hybrid genetic algorithm with adaptive local search scheme for solving multistage-based supply chain problems," Computers and Industrial Engineering, vol. 56, no. 3, pp. 821-838, 2009.

[26] F. Sivrikaya-Şrifoğlu and G. Ulusoy, "Parallel machine scheduling with earliness and tardiness penalties," Computers and Operations Research, vol. 26, no. 8, pp. 773-787, 1999.

[27] L. Min and W. Cheng, "A genetic algorithm for minimizing the makespan in the case of scheduling identical parallel machines," Artificial Intelligence in Engineering, vol. 13, no. 4, pp. 399-403, 1999.

[28] J. M. Garcia, S. Lozano, K. Smith, T. Kwok, and G. Villa, "Coordinated scheduling of production and delivery from multiple plants and with time windows using genetic algorithms," in Proceedings of the 9th International Conference on Neural Information Processing, vol. 3, pp. 1153-1158, Singapore, 2002.

[29] C. W. Feng, T. M. Cheng, and H. T. Wu, "Optimizing the schedule of dispatching RMC trucks through genetic algorithms," Automation in Construction, vol. 13, no. 3, pp. 327-340, 2004.

[30] Y. H. Lee, C. S. Jeong, and C. Moon, "Advanced planning and scheduling with outsourcing in manufacturing supply chain," Computers and Industrial Engineering, vol. 43, no. 1-2, pp. 351-374, 2002.

[31] S. Karabuk, "Modeling and optimizing transportation decisions in a manufacturing supply chain," Transportation Research Part E: Logistics and Transportation Review, vol. 43, no. 4, pp. 321-337, 2007.

[32] S. H. Zegordi, I. N. K. Abadi, and M. A. B. Nia, "A novel genetic algorithm for solving production and transportation 
scheduling in a two-stage supply chain," Computers and Industrial Engineering, vol. 58, no. 3, pp. 373-381, 2010.

[33] R. Chidambaram and D. Armbruster, "Application of genetic algorithms to semiconductor supply chain planning," in Proceedings of the International Conference on Algorithmic Mathematics and Computer Science (AMCS '05), pp. 77-83, Las Vegas, Nev, USA, June 2005.

[34] D. Naso, M. Surico, B. Turchiano, and U. Kaymak, "Genetic algorithms for supply-chain scheduling: a case study in the distribution of ready-mixed concrete," European Journal of Operational Research, vol. 177, no. 3, pp. 2069-2099, 2007.

[35] L. Dong and H. Ding, "A genetic algorithm based dynamic berth allocation strategy for container terminals," in Proceedings of the 8th International Conference of Chinese Logistics and Transportation Professionals, Chengdu, China, August 2008.

[36] M. R. Delavar, M. Hajiaghaei-Keshteli, and S. Molla-AlizadehZavardehi, "Genetic algorithms for coordinated scheduling of production and air transportation," Expert Systems with Applications, vol. 37, no. 12, pp. 8255-8266, 2010.

[37] C. L. Yang and Y. K. Chen, "Using genetic algorithm for better route arrangement a case of Taiwan pelican express company," in Proceedings of the IEEE International Conference on Service Operations and Logistics, and Informatics (SOLI '06), pp. 669673, Shanghai, China, June 2006.

[38] S. J. Sadjadi, M. Jafari, and T. Amini, "A new mathematical modeling and a genetic algorithm search for milk run problem (an auto industry supply chain case study)," International Journal of Advanced Manufacturing Technology, vol. 44, no. 12, pp. 194-200, 2009.

[39] M. Azarmi, A. Jahanmiri, and S. Vakili, "An optimization model for refinery production scheduling (design a software using genetic algorithms \& MILP)," in Proceedings of the 8th World Congress of Chemical Engineering, August 2009.

[40] H. C. W. Lau, T. M. Chan, W. T. Tsui, and W. K. Pang, "Application of genetic algorithms to solve the multidepot vehicle routing problem," IEEE Transactions on Automation Science and Engineering, vol. 7, no. 2, Article ID 4840417, pp. 383-392, 2010.

[41] V. Chankong and Y. Y. Haimes, Multiobjective Decision Making Theory and Methodology, Elsevier, New York, NY, USA, 1983.

[42] F. T. S. Chan and S. H. Chung, "A multi-criterion genetic algorithm for order distribution in a demand driven supply chain," International Journal of Computer Integrated Manufacturing, vol. 17, no. 4, pp. 339-351, 2004.

[43] F. T. S. Chan, S. H. Chung, and S. Wadhwa, "A hybrid genetic algorithm for production and distribution," Omega, vol. 33, no. 4, pp. 345-355, 2005.

[44] K. Al-Mutawah, V. Lee, and Y. Cheung, "Modeling supply chain complexity using a distributed multi-objective genetic algorithm," in Proceedings of International Conference on Computational Science and Its Applications, vol. 3980 of Lecture Notes in Computer Science, pp. 586-595, Melbourne, Australia, 2006.

[45] F. Altiparmak, M. Gen, L. Lin, and T. Paksoy, "A genetic algorithm approach for multi-objective optimization of supply chain networks," Computers and Industrial Engineering, vol. 51, no. 1, pp. 196-215, 2006.

[46] R. Z. Farahani and M. Elahipanah, "A genetic algorithm to optimize the total cost and service level for just-in-time distribution in a supply chain," International Journal of Production Economics, vol. 111, no. 2, pp. 229-243, 2008.

[47] Z. H. Che and C. J. Chiang, "A modified Pareto genetic algorithm for multi-objective build-to-order supply chain planning with product assembly," Advances in Engineering Software, vol. 41, no. 7-8, pp. 1011-1022, 2010.

[48] A. Gunasekaran and E. W. T. Ngai, "Modeling and analysis of build-to-order supply chains," European Journal of Operational Research, vol. 195, no. 2, pp. 319-334, 2009.

[49] D. Fogel and K. Chellapilla, "Revisiting evolutionary programming," in Applications and Science of Computational Intelligence, vol. 3390, pp. 2-11, Orlando, Fla, USA, March 1998.

[50] X. Y. Huang and Z. Lu, "Interactive evolutionary programming and its application in supply chain," Dongbei Daxue Xuebao/Journal of Northeastern University, vol. 21, no. 5, pp. 569-572, 2000.

[51] J. Li, J. R. Wang, Z. W. Hu, and J. H. Zhang, "EP-based optimization of strategic safety stocks in reverse logistics systems," Journal of Dong Hua University, vol. 21, no. 5, pp. 65-68, 2004.

[52] J. Homberger, "A generic coordination mechanism for lotsizing in supply chains," Electronic Commerce Research, vol. 11, no. 2, pp. 1123-149, 2011.

[53] V. Dalkilic, E. J. Lodree Jr., A. Ramesh, and G. Sarpkaya, "Order splitting for multiple-supplier multiple-item healthcare supply chains," in Proceedings of the IIE Annual Conference and Exhibition, Orlando, Fla, USA, 2006.

[54] M. A. Falcone, H. S. Lopes, and L. Dos Santos Coelho, "Supply chain optimisation using evolutionary algorithms," International Journal of Computer Applications in Technology, vol. 31, no. 3-4, pp. 158-167, 2008.

[55] S. Routroy and R. Kodali, "Differential evolution algorithm for supply chain inventory planning," Journal of Manufacturing Technology Management, vol. 16, no. 1, pp. 7-17, 2005.

[56] S. Routroy and P. Sanisetty, "Inventory planning for a multiechelon supply chain," International Journal of Operational Research, vol. 2, no. 3, pp. 269-283, 2007.

[57] S. Routroy and K. C. Maddala, "Multi-echelon supply chain inventory planning with demand and leadtime uncertainty," International Journal of Operational Research, vol. 5, no. 3, pp. 251-264, 2009.

[58] K. Prasertwattana and Y. Shimizu, "Optimization of material ordering and inventory control of supply chain through an incentive scheme using differential evolution," Journal of Japan Industrial Management Association, vol. 59, no. 4, pp. 283-289, 2008.

[59] B. V. Babu and A. M. Gujarathi, "Multi-objective differential evolution (MODE) for optimization of supply chain planning and management," in Proceedings of the IEEE Congress on Evolutionary Computation (CEC '07), pp. 2732-2739, Singapore, September 2007.

[60] L. Dos Santos Coelho and H. S. Lopes, "Supply chain optimization using chaotic differential evolution method," in Proceedings of the IEEE International Conference on Systems, Man and Cybernetics, pp. 3114-3119, Montréal, Canada, October 2006.

[61] J.-M. Xu, J.-Z. Xiong, Y. Chen, and G.-W. Hu, "Supply chain optimization using migration differential evolution ensemble," in Proceedings of the IEEE International Conference on Intelligent Computing and Intelligent Systems (ICIS '10), vol. 3, pp. 755-759, Xiamen, China, 2010.

[62] A. Colorni, M. Dorigo, and V. Maniezzo, "Distributed optimization by ant colonies," in Proceedings of the European Conference on Artificial Life, pp. 134-142, Paris, France, 1991.

[63] C. A. Silva, J. M. C. Sousa, T. A. Runkler, and J. M. G. S. Da Costa, "Distributed optimisation of a logistic system and its suppliers using ant colonies," International Journal of Systems Science, vol. 37, no. 8, pp. 503-512, 2006. 
[64] C. A. Silva, J. M. C. Sousa, T. A. Runkler, and J. M. G. Sá da Costa, "Distributed supply chain management using ant colony optimization," European Journal of Operational Research, vol. 199, no. 2, pp. 349-358, 2009.

[65] H. S. Wang, "A two-phase ant colony algorithm for multiechelon defective supply chain network design," European Journal of Operational Research, vol. 192, no. 1, pp. 243-252, 2009.

[66] L. A. Moncayo-Martínez and D. Z. Zhang, "Multi-objective ant colony optimisation: a meta-heuristic approach to supply chain design," International Journal of Production Economics, vol. 131, no. 1, pp. 407-420, 2011.

[67] M. Clerc and J. Kennedy, "The particle swarm-explosion, stability, and convergence in a multidimensional complex space," IEEE Transactions on Evolutionary Computation, vol. 6, no. 1, pp. 58-73, 2002.

[68] J. Izquierdo, R. Minciardi, I. Montalvo, M. Robba, and M. Tavera, "Particle Swarm Optimization for the biomass supply chain strategic planning," in Proceedings of the International Congress on Environmental Modelling and Software, pp. 12721280, 2008.

[69] G. W. Hu, "Generalized genetic particle swarm optimization for supply chain optimization," Journal of Computer Applications, vol. 28, no. 11, pp. 2840-2843, 2008.

[70] R. Kadadevaramath, K. Mohanasundaram, K. Rameshkumar, and B. Chandrashekhar, "Production and distribution scheduling of Supply Chain structure using intelligent Particle Swarm Optimisation algorithm," International Journal of Intelligent Systems Technologies and Applications, vol. 6, no. 34, pp. 249-268, 2009.

[71] M. Bachlaus, M. K. Pandey, C. M. Shankar, and M. K. Tiwari, "Designing an integrated multi-echelon agile supply chain network: a Hybrid Taguchi-Particle swarm optimization approach," International Journal of Flexible Manufacturing System, vol. 19, no. 6, pp. 486-515, 2007.

[72] A. A. Taleizadeh, S. T. A. Niaki, N. Shafii, R. G. Meibodi, and A. Jabbarzadeh, "A particle swarm optimization approach for constraint joint single buyer-single vendor inventory problem with changeable lead time and (r,Q) policy in supply chain," International Journal of Advanced Manufacturing Technology, vol. 51, no. 9-12, pp. 1209-1223, 2010.

[73] A. K. Sinha, H. K. Aditya, M. K. Tiwari, and F. T. S. Chan, "Agent oriented petroleum supply chain coordination: coevolutionary particle swarm optimization based approach," Expert Systems with Applications, vol. 38, no. 4, pp. 6132-6145, 2011.

[74] X. J. Wu, H. Zhou, and C. H. Liang, "Collaborative optimization of multi-echelon supply chain based on co-evolutionary particle swarm optimization," Computer Integrated Manufacturing Systems, vol. 16, no. 1, pp. 127-132, 2010.

[75] G. Taguchi, S. Chowdhary, and S. Taguchi, Robust engineering, McGraw-Hill, New York, NY, USA, 2000.

[76] C. Soares, G. Dozier, E. Lodree, J. Phillips, K. Nobles, and W. P. Yong, "Optimization of the multiple retailer supply chain management problem," in Proceedings of the 46th Annual Southeast Regional Conference on XX ACM-SE, pp. 490-495, Auburn, Ala, USA, March 2008.

[77] X. S. Yang, "Engineering optimizations via nature-inspired virtual bee algorithms," in Artificial Intelligence and Knowledge Engineering Applications: A Bioinspired Approach, vol. 3562 of Lecture Notes in Computer Science, pp. 317-323, Springer, Berlin, Germany, 2005.
[78] D. T. Pham, A. Ghanbarzadeh, E. Koc, S. Otri, S. Rahim, and M. Zaidi, "The bees algorithm," Tech. Rep., Manufacturing Engineering Centre, Cardiff University, 2005.

[79] D. Karaboga, "An idea based on honeybee swarm for numerical optimization,” Tech. Rep. TR06, Erciyes University, 2005.

[80] D. Karaboga and B. Basturk, "A powerful and efficient algorithm for numerical function optimization: artificial bee colony (ABC) algorithm," Journal of Global Optimization, vol. 39, no. 3, pp. 459-471, 2007.

[81] V. V. Kumar, F. T. S. Chan, N. Mishra, and V. Kumar, "Environmental integrated closed loop logistics model: an artificial bee colony approach," in Proceedings of the 8th International Conference on Supply Chain Management and Information Systems: Logistics Systems and Engineering (SCMIS '10), Hong Kong, China, 2010.

[82] A. Pal, F. T. S. Chan, B. Mahanty, and M. K. Tiwari, "Aggregate procurement, production, and shipment planning decision problem for a three-echelon supply chain using swarm-based heuristics," International Journal of Production Research, vol. 49, no. 10, pp. 2873-2905, 2011.

[83] S. Banerjee, G. S. Dangayach, S. K. Mukherjee, and P. K. Mohanti, "Modelling process and supply chain scheduling using hybrid meta-heuristics," Studies in Computational Intelligence, vol. 128, pp. 277-300, 2008.

[84] J.-Q. Li, Q.-K. Pan, and K.-Z. Gao, "Pareto-based discrete artificial bee colony algorithm for multi-objective flexible job shop scheduling problems," International Journal of Advanced Manufacturing Technology, vol. 55, no. 9-12, pp. 1159-1169, 2011.

[85] A. Prakash and S. G. Deshmukh, "A multi-criteria customer allocation problem in supply chain environment: an artificial immune system with fuzzy logic controller based approach," Expert Systems with Applications, vol. 38, no. 4, pp. 3199-3208, 2011.

[86] M. Shukla, N. Shukla, M. K. Tiwari, and F. T. S. Chan, "Integrated model for the batch sequencing problem in a multi-stage supply chain: an artificial immune system based approach," International Journal of Production Research, vol. 47, no. 4, pp. 1015-1037, 2009.

[87] M. Hajiaghaei-Keshteli, "The allocation of customers to potential distribution centers in supply chain networks: GA and AIA approaches," Applied Soft Computing Journal, vol. 11, no. 2, pp. 2069-2078, 2011.

[88] D. Srinivasan and T. H. Seow, "Particle swarm inspired evolutionary algorithm (PS-EA) for multi-objective optimization problem," in Proceedings of the IEEE Congress on Evolutionary Computation, pp. 2292-2297, Canbella, Australia, 2003.

[89] R. Poli, C. Di Chio, and W. B. Langdon, "Exploring extended particle swarms: a genetic programming approach," in Proceedings of the Genetic and Evolutionary Computation Conference (GECCO '05), pp. 169-176, Washington, DC, USA, June 2005.

[90] W. Fu, M. Johnston, and M. Zhang, "Hybrid particle swarm optimisation algorithms based on differential evolution and local search," in Proceedings of the Australasian Conference on Artificial Intelligence, vol. 6464 of Lecture Notes in Artificial Intelligence, pp. 313-322, 2010.

[91] Y. J. Wang, "Improving particle swarm optimization performance with local search for high-dimensional function optimization," Optimization Methods and Software, vol. 25, no. 5, pp. 781-795, 2010.

[92] S. Meeran and M. S. Morshed, "A hybrid genetic tabu search algorithm forsolving job shop scheduling problems: a case study," Journal of Intelligent Manufacturing. In press. 
[93] X. Wang, X. Z. Gao, and S. J. Ovaska, "A hybrid artificial immune optimization method," International Journal of Computational Intelligence Systems, vol. 2, no. 3, pp. 249-256, 2009.

[94] D. Guo, J. Wang, J. Huang, R. Han, and M. Song, "ChaoticNSGA-II: an effective algorithm to solve multi-objective optimization problems," Proceedings of the International Conference on Intelligent Computing and Integrated Systems (ICISS '10), pp. 20-23, 2010.

[95] R. Caponetto, L. Fortuna, S. Fazzino, and M. G. Xibilia, "Chaotic sequences to improve the performance of evolutionary algorithms," IEEE Transactions on Evolutionary Computation, vol. 7, no. 3, pp. 289-304, 2003.

[96] G. Zhang, "Quantum-inspired evolutionary algorithms: a survey and empirical study," Journal of Heuristics, vol. 17, no. 3, pp. 303-351, 2010.

[97] E. G. Bakhoum and C. Toma, "Specific mathematical aspects of dynamics generated by coherence functions," Mathematical Problems in Engineering, vol. 2011, Article ID 436198, 10 pages, 2011.

[98] E. G. Bakhoum and C. Toma, "Dynamical aspects of macroscopic and quantum transitions due to coherence function and time series events," Mathematical Problems in Engineering, vol. 2010, Article ID 428903, 13 pages, 2010.

[99] F. Tao, L. Zhang, Z. H. Zhang, and A. Y. C. Nee, "A quantum multi-agent evolutionary algorithm for selection of partners in a virtual enterprise," Manufacturing Technology, vol. 59, no. 1, pp. 485-488, 2010. 


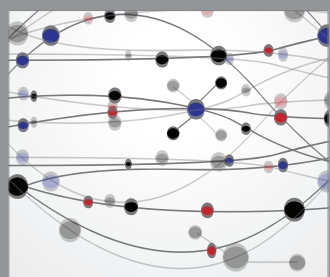

The Scientific World Journal
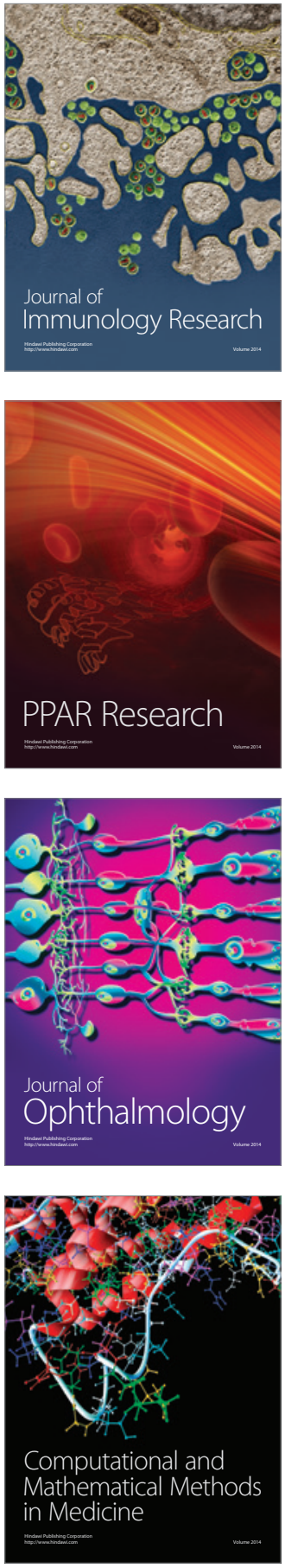

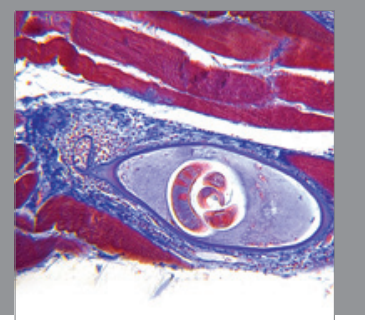

Gastroenterology

Research and Practice
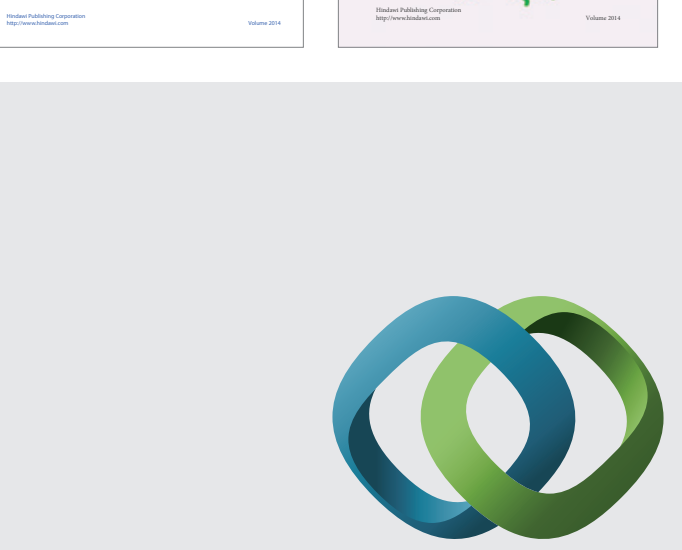

\section{Hindawi}

Submit your manuscripts at

http://www.hindawi.com
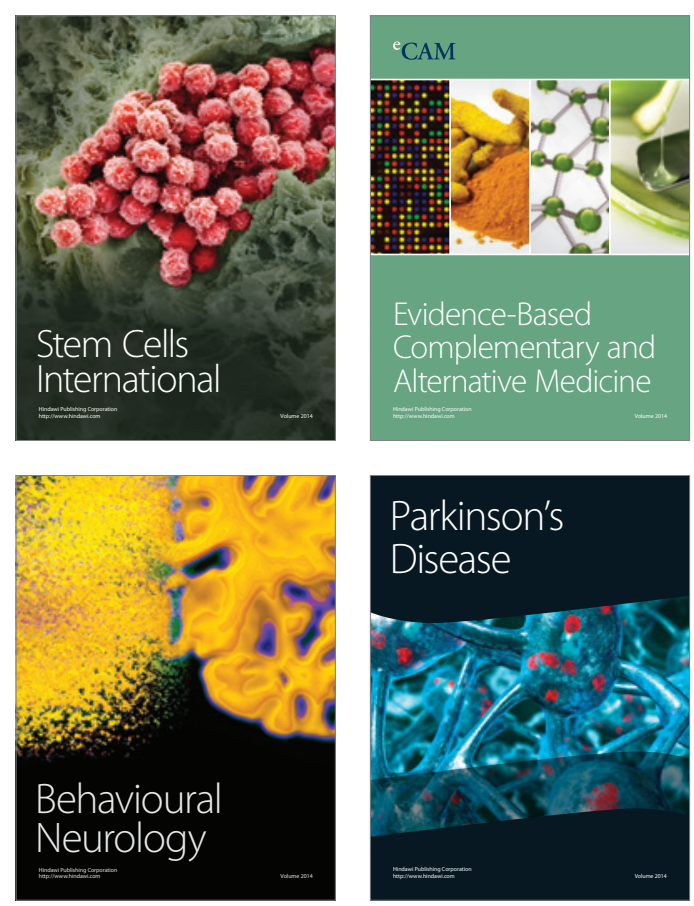

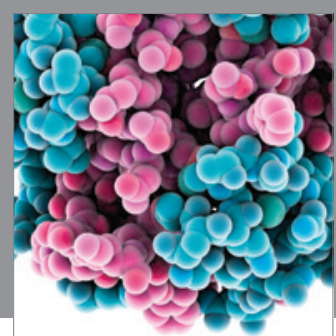

Journal of
Diabetes Research

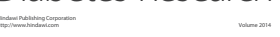

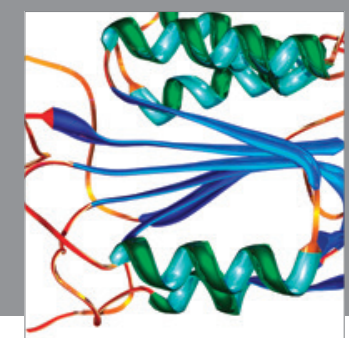

Disease Markers
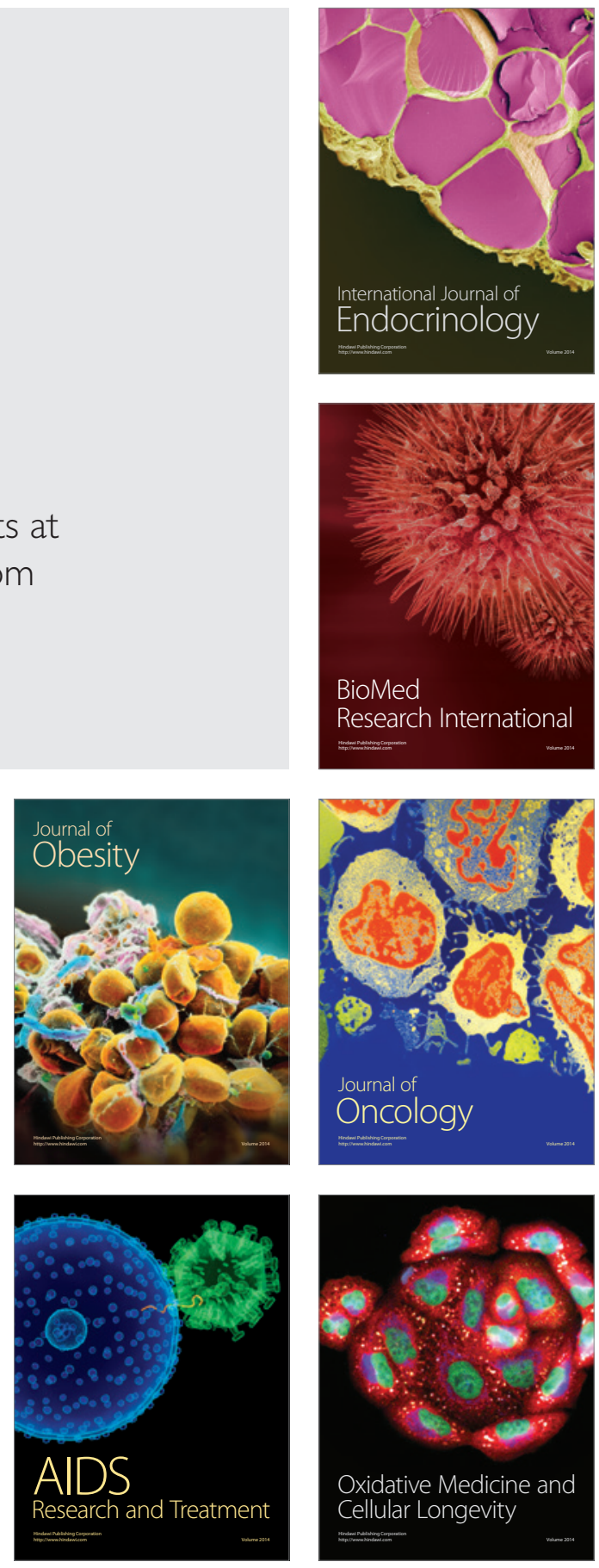